\title{
Cultivo mixto de especies forestales de turno corto (Robinia pseudoacacia y Populus $x$ euroamericana clon AF2) con fines energéticos
}

\author{
Alesso Oviedo, S.P. ${ }^{*}$, Alaejos Gutiérrez, J. ${ }^{1}$, Tapias Martín, R. ${ }^{1}$, \\ Fernández Martínez, M. ${ }^{1}$ \\ ${ }^{1}$ Universidad de Huelva. Departamento de Ciencias Agroforestales, Escuela Técnica Superior de Ingeniería. \\ Campus La Rábida S/N, 21819, Palos de La Frontera (Huelva) \\ *Autor para correspondencia: silviapatricia.alesso@alu.uhu.es
}

\section{Resumen}

El uso de fuentes de energía limpia y renovable, como la biomasa lignocelulósica, puede ayudar a paliar el efecto sobre el cambio climático, reducir la contaminación y la dependencia energética, así como ayudar a recuperar terrenos degradados y a potenciar las economías rurales. En este trabajo se realizaron ensayos, durante cuatro años, de cultivos mixtos para la producción de biomasa en una zona de clima mediterráneo continental de inviernos fríos, utilizando Robinia pseudoacacia, especie fijadora de $\mathrm{N}_{2}$, y Populus x euroamericana clon AF2. La fijación natural del $\mathrm{N}_{2}$ atmosférico puede ayudar a disminuir las necesidades de fertilización nitrogenada de las plantaciones. Las dos especies se mezclaron en distintas proporciones. El chopo fue más productivo en biomasa que la falsa acacia los dos primeros años pero, posteriormente, fue alcanzado por ésta. A pesar de que el chopo presentó mayor diámetro y altura, la baja densidad de la madera hizo que no destacase en biomasa. La producción media de biomasa seca leñosa aérea fue de $3.5 \mathrm{tha}^{-1}$ el primer año, pero aumentó a 12-19 t ha ${ }^{-1}$ año $^{-1}$ los dos años siguientes. La falsa acacia superó mejor que el chopo las épocas de mayor estrés hídrico, cuando el riego no llegó a ser suficiente. Tras cuatro años de cultivo y a pesar de la extracción de biomasa, que en el cuarto año promedió $38.7 \mathrm{t} \mathrm{ha}^{-1}$, los parámetros de fertilidad del suelo no empeoraron sino que mostraron tendencia a permanecer constantes o a mejorar.

Palabras clave: Biomasa, cultivos energéticos, fijación $\mathrm{N}_{2}$, chopo, falsa acacia 


\section{Introducción}

Los compromisos internacionales adquiridos por España y la UE obligan a impulsar el uso de fuentes de energía limpia y renovable para paliar el efecto sobre el cambio climático y reducir la contaminación (Consejo de Europa, 2007; Naciones Unidas, 2015). En este contexto, la biomasa lignocelulósica se presenta como una fuente de energía renovable, con aplicaciones térmicas y eléctricas que, además, puede ayudar a recuperar terrenos agrícolas degradados y a potenciar las economías rurales. La estrategia española contra el cambio climático, no solo pretende potenciar el uso de cultivos energéticos cuyos ciclos energéticos y de emisiones $\mathrm{CO}_{2}$ sean positivos, sino también que se reduzca el uso de fertilizantes nitrogenados (IDAE, 2007; Tapias et al., 2008). No obstante, la falta de incentivos suficientes en España, y aspectos coyunturales como la caída del precio del petróleo, han dado lugar a que aún no se haya generalizado la implantación de un sector empresarial que verdaderamente los potencie para uso energético, mediante ley de oferta-demanda, salvo en casos muy concretos (p.ej. plantaciones de especies leñosas hechas por la empresa ENCE), a pesar de llevar más de 20 años realizando experiencias con cultivos leñosos. Es de esperar que el nuevo impulso que ha dado la Conferencia de las Partes de la Convención Marco sobre el Cambio Climático de la ONU, celebrada en París (Naciones Unidas, 2015) sirva de estímulo a la iniciativa privada y al apoyo institucional.

Uno de los aspectos menos ensayados es el uso de cultivos energéticos mixtos en el que se utilicen dos o más especies en determinadas proporciones, de tal forma que se complementen entre sí, buscando incrementar la cantidad y calidad de la biomasa producida (Forrester et al., 2006; Laclau et al., 2008) y/o mejorar a medio plazo la fertilidad del suelo (Pereira et al., 2011). En las últimas décadas se han ensayado combinaciones de especies leñosas de igual o distinto potencial de crecimiento y mezclas de árboles con cultivos herbáceos (Little et al., 2002), enfocados a la producción de biomasa con fines energéticos.

Los cultivos mixtos pueden afectar a la producción final por modificar la relación de competencia, la velocidad de descomposición de los restos, la fertilidad de los suelos y posiblemente reducir los daños de enfermedades y plagas (Forrester et al., 2004; Kelty, 2006; Grünewald et al., 2009; Pereira et al., 2011). Presentan ventajas e inconvenientes. Por ejemplo, en cultivo mixto de una especie arbórea (eucalipto) con herbáceas se comprobó que la competencia de las herbáceas redujo el crecimiento de los árboles, no obstante esta reducción resultó menor cuando aquellas eran leguminosas (Marques et al., 2015). Asimismo, en el caso de mezclar una especie de crecimiento muy rápido con otra que no lo es, la segunda podría quedar dominada y suprimida (Bauhus et al., 2000; Kelty, 2006; Forrester et al., 2006).

Estudios sobre las especies más empleadas en plantaciones han demostrado un incremento de las necesidades de $\mathrm{N}$ con las rotaciones (Gonçalves et al., 2004; Laclau et al., 2005). La fertilización con $\mathrm{N}$ es la más costosa en el balance económico del cultivo y los efectos ambientales de su aplicación deben ser estudiados con mayor profundidad (Fisher and Binkley, 2000). Asimismo, el coste energético de la fabricación 
de fertilizantes es el más alto de todos los procesos de cultivo (McLaughlin et al., 2000; IDAE, 2007). Por ello, el empleo de especies fijadoras de $\mathrm{N}_{2}$ en un cultivo mixto y de crecimiento rápido podría ayudar a reducir la fertilización nitrogenada sin renunciar a la productividad (Parrotta, 1999; Voigtlaender et al., 2012). En todo caso, se deben valorar aspectos como el balance económico y ambiental en varios ciclos, la facilidad de la cosecha y la aptitud de la biomasa de las distintas especies para el aprovechamiento energético (Laclau et al., 2008).

Muchas especies arbóreas forestales son adecuadas para la producción de biomasa en turnos cortos. Entre ellas, en zonas templadas de inviernos fríos, podemos destacar a Robinia pseudoacacia y al género Populus. Éste último tiene una gran capacidad de adaptación a distintas condiciones medioambientales, alta capacidad de hibridación y facilidad para multiplicarse vegetativamente, aunque suele requerir humedad edáfica alta. Estas características, unidas a su rápido crecimiento, han contribuido al desarrollo de una oferta clonal muy extensa, que precisa de una selección previa para cada sitio y uso en concreto (Sixto et al., 2007; Sixto et al., 2015). El cultivo de $R$. pseudoacacia es una alternativa económicamente competitiva por su gran tolerancia a la sequía, su capacidad de fijación de $\mathrm{N}_{2}$ y su notable productividad tratada a turnos cortos (Grünewald et al., 2009). Por tanto, resultaría de interés analizar el uso de estos dos taxones en cultivo mixto, para determinar el grado de complementación que pueden establecer entre ellos.

\section{Objetivos}

El objetivo de este trabajo es evaluar durante los primeros cuatro años, el crecimiento, la producción de materia seca aérea y el efecto sobre el suelo de dos taxones leñosos en cultivo mixto, Robinia pseudoacacia y Populus x euroamericana clon "AF2", de interés para cultivos con fines energéticos, así como analizar los efectos que provoca la plantación mixta de las dos diferentes especies en la producción de biomasa total.

\section{Metodología}

\subsection{Material vegetal y diseño experimental}

En este estudio se analizó el cultivo de dos taxones arbóreos, Populus $x$ euroamericana (clon “AF2") y Robinia pseudoacacia (AF y Rps, respectivamente). Para el chopo se utilizaron estacas de madera dura, de $20-25 \mathrm{~cm}$ de longitud, mientras que para Rps se utilizaron plantas de vivero a raíz desnuda de $40-60 \mathrm{~cm}$ de altura. Los dos taxones se plantaron en cinco tratamientos, considerando cultivo puro o mezclado en distintas proporciones: R4AF0 (100\% Rps), R3AF1 (75\% Rps y $25 \% \mathrm{AF}), \mathrm{R} 2 \mathrm{AF} 2(50 \%$ Rps y $50 \% \mathrm{AF}), \mathrm{R} 1 \mathrm{AF} 3(25 \% \mathrm{Rps}$ y $75 \% \mathrm{AF})$ y $\mathrm{R} 0 \mathrm{AF} 4$ $(100 \% \mathrm{AF})$. 
La plantación se realizó a finales de marzo de 2011, sobre un terreno agrícola llano (Huéscar, Granada, UTM, Zona 30S, X: 535459 E, Y: 4191516 N, 1085 msnm). El suelo es calizo, de textura franco-arcillosa, permeable, con $2.9 \% \mathrm{MO}$, $\mathrm{pH}=8.3,12 \%$ de caliza activa, bajo clima mediterráneo continental de inviernos fríos, con $14.0^{\circ} \mathrm{C}$ y $400 \mathrm{~mm}$ de temperatura y precipitación medias anuales. Los registros meteorológicos durante el período de estudio se muestran en la Fig. 1. Antes de la plantación se aplicó estiércol de oveja en la dosis de 25 t ha ${ }^{-1}$. Como algo más del $75 \%$ de las plantas de Robinia presentaron síntomas de clorosis foliar, se aplicó quelato de hierro (EDDHA 6\%, FerroActiv ${ }^{\circledR}, 2.5 \mathrm{~kg} \mathrm{ha}^{-1}$ año $^{-1}$ ) para intentar paliarla, pero no se logró del todo. Los dos primeros años de cultivo no se añadió fertilizante adicional, pero el tercer año se añadió $250 \mathrm{~kg} \mathrm{ha}^{-1}$ de fertilizante granulado 15-15-15 (20S) (Fertiberia), aplicado al suelo a finales de marzo. No se pretendía realizar una fertilización óptima, para no enmascarar el posible efecto sobre la producción de la especie fijadora de $\mathrm{N}_{2}$, incluida en el ensayo. No obstante, no se aplicaron tratamientos diferenciados de fertilización.

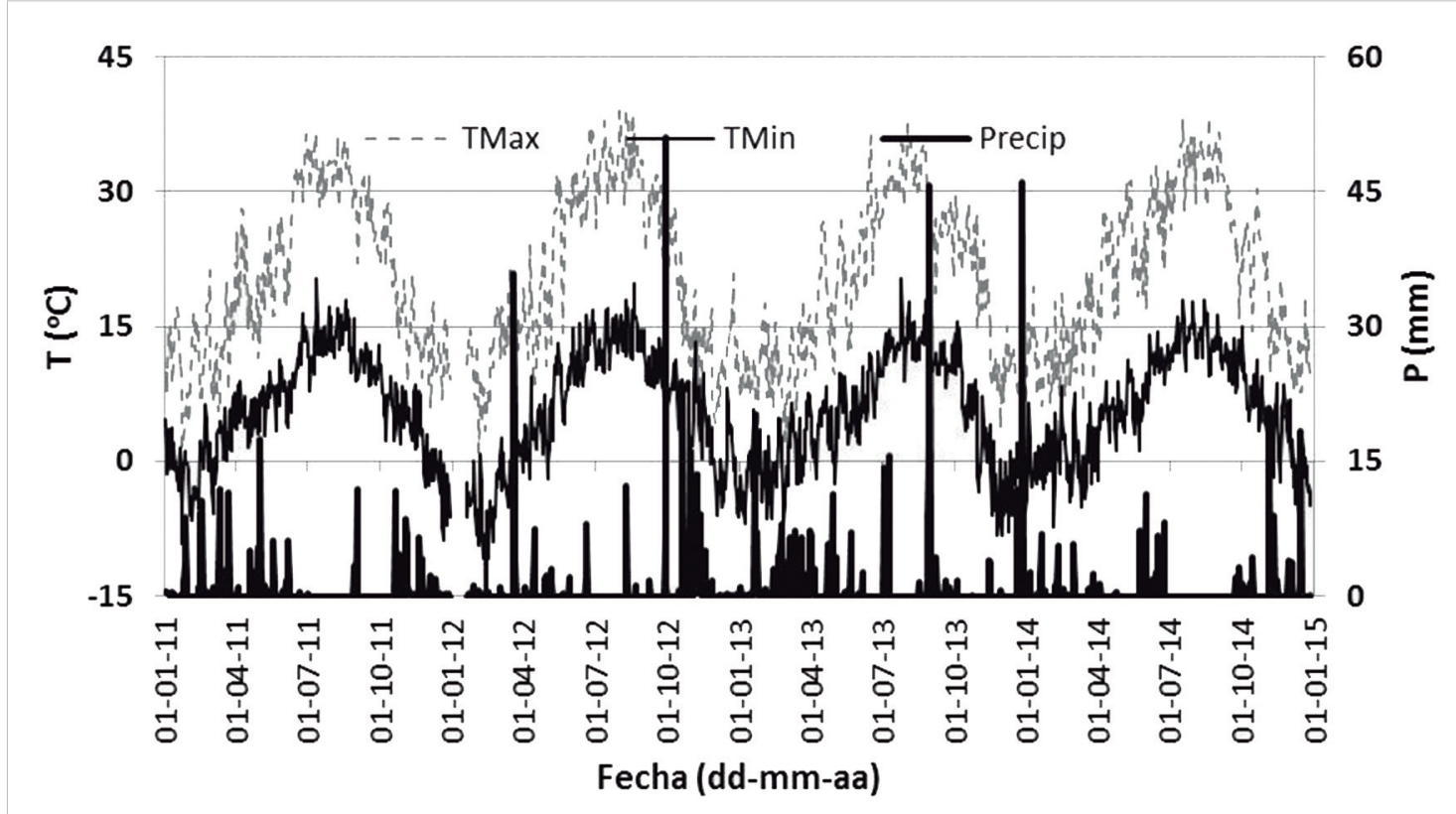

Figura 1. Evolución temporal de las temperatura máximas y mínima $\left(\mathrm{T},{ }^{\circ} \mathrm{C}\right)$ y de las precipitaciones $(\mathrm{P}, \mathrm{mm})$ diarias registradas en la parcela de ensayo y durante el período de estudio (años 2011 a 2015).

El diseño experimental consistió en cuatro bloques completos aleatorizados donde se dispusieron los dos taxones mencionados. La parcela elemental, de 48 plantas, consistió en tres filas de 16 plantas cada una, con separación entre filas de $3 \mathrm{~m}$ y separación entre plantas dentro de una misma fila de $0.6 \mathrm{~m}$ (5556 plantas/ha). Se consideró una parcela elemental por bloque. La parcela contó con riego de apoyo para la época estival (junio-septiembre) mediante líneas de goteros a lo largo de las filas. Tras dos períodos vegetativos completos, en marzo de 2013, se cortaron la mitad de los 
bloques para analizar la capacidad de rebrote de las dos especies. A partir de esta fecha se diferenció el crecimiento entre plantas cortadas y sin cortar. Los tres primeros años de cultivo se procuró que la aportación anual de agua para las plantas (precipitación + riego) estuviera entre 700 y $800 \mathrm{~mm}$, con ello finalizaba el turno de tres años previsto. Seguidamente se continuó un año más, reduciendo la dosis de riego, para que el conjunto precipitación+riego fuese aproximadamente la mitad, con el fin de analizar la respuesta de las plantas a un eventual período de sequía (Tab. 1). Esto último provocó el desprendimiento del $50 \%$ de las hojas en los chopos durante el verano del cuarto año, mientras que Rps conservó todas sus hojas.

Tabla 1. Datos meteorológicos registrados durante el estudio. Se presentan los datos para los períodos de tiempo comprendidos entre 1-octubre y 30-septiembre de cada año hidrológico, incluyendo, por tanto, un período de reposo invernal y uno vegetativo en cada caso. Datos tomados de la estación meteorológica de Puebla de Don Fadrique (Granada) de coordenadas UTM, Zone 30 S, X: 554375 E, Y: 4192250 N, 1110 msnm.

\begin{tabular}{lccccccc}
\hline Período & $\begin{array}{c}\text { TMax } \\
\left({ }^{\circ} \mathrm{C}\right)\end{array}$ & $\begin{array}{c}\text { TmedMax } \\
\left({ }^{\circ} \mathrm{C}\right)\end{array}$ & $\begin{array}{c}\text { TMin } \\
\left({ }^{\circ} \mathrm{C}\right)\end{array}$ & $\begin{array}{c}\text { TmedMin } \\
\left({ }^{\circ} \mathrm{C}\right)\end{array}$ & $\begin{array}{c}\text { Tmed } \\
\left({ }^{\circ} \mathrm{C}\right)\end{array}$ & $\begin{array}{c}\mathrm{P} \\
(\mathrm{mm})\end{array}$ & $\begin{array}{c}\text { Riego } \\
(\mathrm{mm})\end{array}$ \\
\hline $2010-11$ & 36.6 & 21.8 & -9.1 & 6.8 & 14.3 & 500 & 350 \\
$2011-12$ & 39.1 & 21.3 & -15.5 & 5.3 & 13.3 & 231.9 & 450 \\
$2012-13$ & 37.6 & 19.1 & -7.0 & 5.4 & 12.2 & 415.3 & 350 \\
$2013-14$ & 38.5 & 21.2 & -8.3 & 5.6 & 13.4 & 159.6 & 250 \\
\hline
\end{tabular}

\subsection{Mediciones}

Desde el momento de la plantación y hasta febrero de 2015 (4 períodos vegetativos) se llevó un seguimiento estacional (cada 2-3 meses hasta la corta del segundo año y cada 6 meses el resto) del crecimiento en altura y diámetro basal, medido este último a 5-7 cm sobre la superficie del suelo. En cada fecha de medición se midieron 10 plantas por taxón y bloque, escogidas aleatoriamente, preferentemente en la fila central para reducir el efecto borde. Asimismo, en el mes de diciembre de cada año se cosecharon 4-6 plantas por taxón para su medición (altura y diámetro), secado en estufa a $80{ }^{\circ} \mathrm{C}$ hasta peso constante y posterior pesaje. Para la determinación del peso seco, se separaron las leñas finas $(<2.5 \mathrm{~cm}$ diámetro $)$ y gruesas $(>2.5 \mathrm{~cm}$ de diámetro). En dichas fechas las plantas no tenían hojas por su condición de caducas. Cabe mencionar que las acacias presentaron su primera floración y fructificación el tercer año de cultivo, siendo estas abundantes el cuarto año. Asimismo se tomaron muestras de suelo $(0-20 \mathrm{~cm})$ antes de la plantación, al momento de esta y al final del estudio, para su oportuno análisis de textura y fertilidad por métodos estándar. 


\subsection{Análisis de datos}

Los datos de las muestras de plantas cosechadas al final de cada año fueron usados para construir relaciones alométricas, mediante modelos de regresión simple o múltiple, para cada taxón, que nos permitieron estimar parámetros cómo biomasa seca total o biomasa seca leñosa en función del diámetro o del diámetro y altura.

El análisis de los parámetros medidos (altura, diámetro, biomasa seca) se realizó para cada fecha por separado y se trató mediante ANOVA de un factor fijo, el tratamiento de cultivo, dentro de un modelo lineal general. El bloque se eliminó del modelo porque su efecto no resultó significativo. No se consideró un ANOVA de medidas repetidas porque las 10 plantas medidas en cada fecha no fueron siempre las mismas. Cuando las diferencias entre los tratamientos resultaron significativas para algún parámetro, la diferenciación entre grupos homogéneos se analizó mediante el test de Tukey HSD o Games-Howell, según se cumpliera o no la igualdad de varianzas. Todas las diferencias significativas se consideraron para un $\alpha=0.05$. El análisis estadístico fue llevado a cabo mediante el software $\operatorname{SPSS}^{\circledast}$ v19.0.

\section{Resultados}

\subsection{Altura de las plantas}

Se obtuvieron diferencias significativas $(p<0.001)$ entre especies, en todas las fechas de medición, para la altura de las plantas. Al final del segundo año el chopo superó en $128 \mathrm{~cm}$ a la falsa acacia ( $505 \pm 6 \mathrm{~cm}$ y $377 \pm 10 \mathrm{~cm}$, respectivamente). Al cuarto, año en las parcelas no cortadas, esta diferencia superó los $2 \mathrm{~m}(652 \pm 4 \mathrm{~cm}$ y $444 \pm 40 \mathrm{~cm}$, respectivamente). La altura media alcanzada en los diferentes tratamientos durante el estudio se muestra en la figura 2. La altura de los árboles del tratamiento R0AF4 (sólo chopo) fue significativamente superior ( $p=0.001$ ) a R4AF0 (sólo Robinia) en todas las fechas. Sin embargo, la altura media de los tratamientos combinados (R1AF3, R2AF2, R3AF1) tomó valores intermedios entre los dos tratamientos puros, no diferenciándose significativamente entre sí ni de los otros dos tratamientos $(p>0.050)$.

\subsection{Diámetro del tronco y producción de biomasa}

El diámetro de la base del tronco principal presentó diferencias significativas ( $p$ $<0.001$ ) entre especies para todas las fechas de medición. Al final del segundo año el chopo superó en $10.62 \mathrm{~mm}$ a la falsa acacia $(49.86 \pm 0.98 \mathrm{~mm}$ y $39.24 \pm 1.37 \mathrm{~mm}$ respectivamente). Al cuarto, año en las parcelas no cortadas, esta diferencia rondó los $20 \mathrm{~mm}(87.18 \pm 1.21 \mathrm{~mm}$ y $68.06 \pm 2.76 \mathrm{~mm}$, respectivamente). Como el diámetro del tronco se correlacionó significativamente con la biomasa de la planta, a efectos de esta publicación vamos a centrarnos en exponer los valores estimados en la produc- 


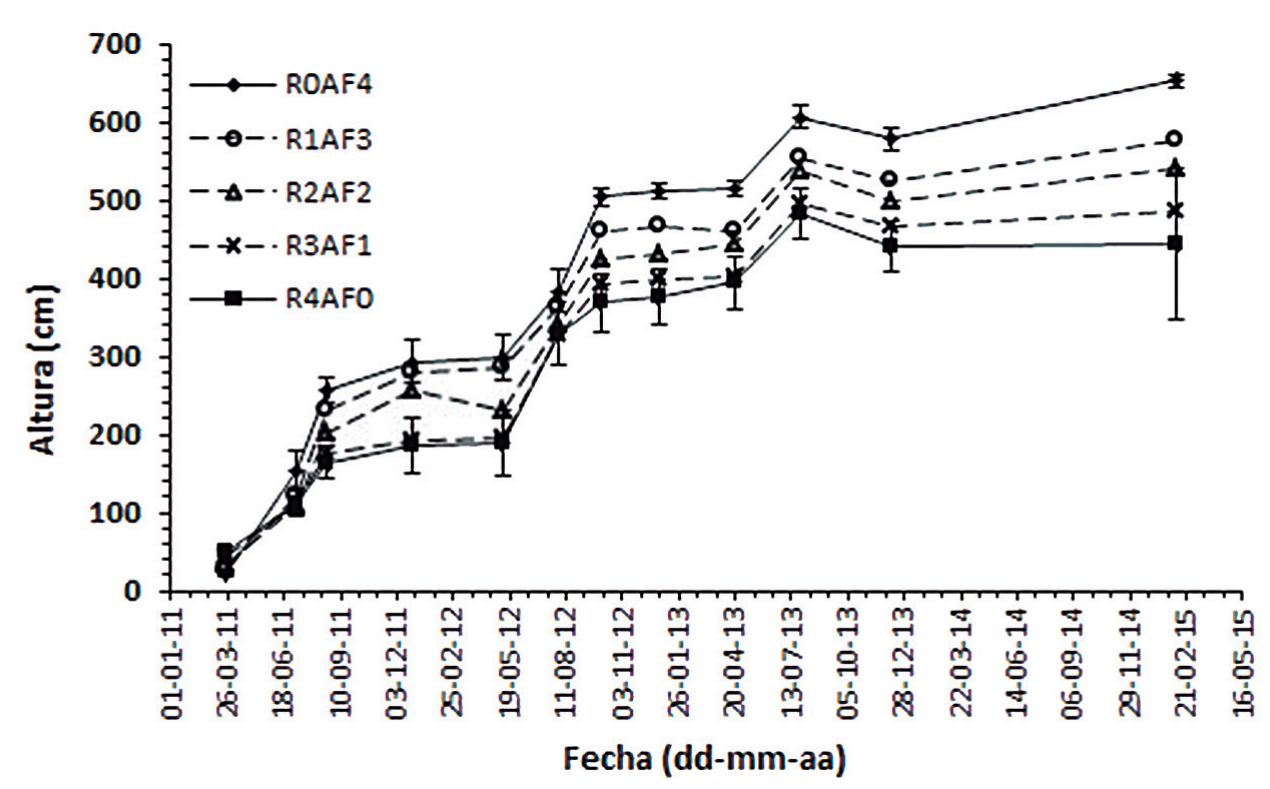

Figura 2. Evolución de la altura de las plantas para los diferentes tratamientos, en los bloques no cortados. Se representa altura media por tratamiento $\mathrm{y}$, en los tratamientos combinados (R1AF3, R2AF2, R3AF1), están incluidos chopos y acacias en conjunto. R0AF4 se diferenció significativamente de R4AF0 en todas las fechas de medición $(p \leq 0.001)$. Los otros tratamientos intermedios no se diferenciaron significativamente de los dos extremos. Solo se muestran las barras de error típico para los dos tratamientos más extremos.

ción de biomasa de los tratamientos ensayados, así como su evolución temporal. De las distintas ecuaciones alométricas analizadas, por su sencillez y alto grado de ajuste, se escogió la de tipo potencial para ambos taxones $\left(B=a \cdot D^{b}\right.$, siendo $B$ la biomasa leñosa seca aérea de la planta, en gramos; y $D$ el diámetro basal, en mm); posteriormente se extrapoló el valor medio de árbol a la hectárea de terreno. Las ecuaciones ajustadas que resultaron para las dos especies fueron: para el chopo, $B=0.0328$ $D^{2.7099}\left(\mathrm{n}=20, \mathrm{R}^{2}=0.98\right)$; mientras que para la Robinia, $B=0.0727 D^{2.6309}\left(\mathrm{n}=16, \mathrm{R}^{2}\right.$ $=0.99)$. Ecuaciones alométricas sencillas, como las aquí presentadas, han sido obtenidas por otros autores (Parrotta, 1999; Paris et al., 2011).

En cuanto a la biomasa seca leñosa aérea, se obtuvieron diferencias significativas $(p<0.001)$ entre las dos especies en el primer año de crecimiento (hasta mayo de 2012) cuando el peso medio de un chopo duplicaba al de la Robinia ( $834 \pm 41$ g y 382 $\pm 31 \mathrm{~g}$, respectivamente). A partir de esta fecha las diferencias entre especies dejaron de ser significativas, a pesar del mayor crecimiento en diámetro y altura del chopo. Para el segundo año el peso medio de un árbol (chopo y Robinia incluidos) fue de $2846 \pm 146 \mathrm{~g}$, el tercer año $6737 \pm 338 \mathrm{~g}$, y en el cuarto año $6966 \pm 387$ g que equivale a $38.7 \mathrm{t} \mathrm{ha}^{-1}$. La biomasa seca estimada y su evolución temporal se muestran en la figura 3. Para el conjunto de tratamientos, la biomasa seca leñosa promedió, aproximadamente, $3.5 \mathrm{tha}^{-1}$ el primer año, $16 \mathrm{t} \mathrm{ha}^{-1}$, en el segundo y $35 \mathrm{t} \mathrm{ha}^{-1}$ el tercero, lo que suponen incrementos anuales de 3.5, 12.5 y $19.0 \mathrm{t} \mathrm{ha}^{-1} \mathrm{año}^{-1}$. El incremento medio durante el $4^{\circ}$ año fue de poco más de $3.5 \mathrm{t} \mathrm{ha}^{-1}$. No se obtuvieron diferencias 


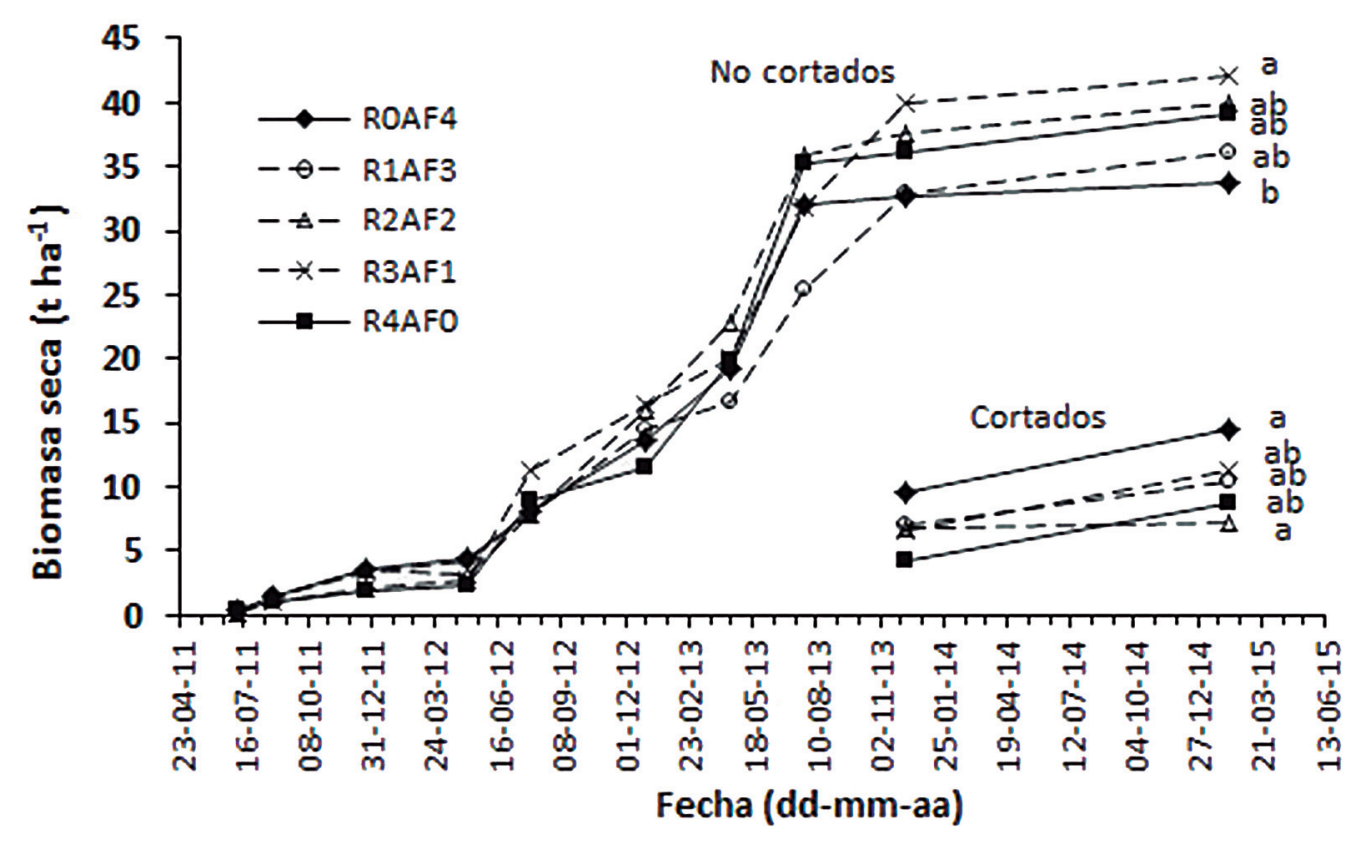

Figura 3. Biomasa leñosa seca producida en las parcelas de cultivos mixtos bajo dos regímenes de corta. La corta se realizó en la mitad de los bloques a los dos años. En la última fecha de medición, letras distintas indican diferencias significativas entre tratamientos. Solo se obtuvieron diferencias significativas en las dos últimas fechas de medición.

significativas entre tratamientos hasta el final del estudio ( $3^{\circ}$ y $4^{\circ}$ años), cuando se observó una tendencia hacia mayores producciones de los tratamientos con mayor proporción de Robinia sobre los de chopo. De hecho, el tratamiento R3AF1 llegó a diferenciarse significativamente del R0AF4 los dos últimos años $(p<0.001$ y $p=$ 0.011 , respectivamente).

El análisis de los rebrotes surgidos de las cepas, tras la corta del segundo año, mostró diferencias significativas en biomasa entre especies $(p=0.001)$ en los dos años siguientes. El chopo superó a la falsa acacia tanto el primer como el segundo año de rebrote $(2731 \pm 309 \mathrm{~g}$ por planta, frente a $1289 \pm 247 \mathrm{~g}$ por planta, al segundo año). Esto supuso que el chopo casi alcanzase $10 \mathrm{tha}^{-1} \mathrm{el}$ primer año y $5 \mathrm{t} \mathrm{ha}^{-1}$ año $\mathrm{o}^{-1}$ más durante el segundo, aún con las restricciones hídricas. Por su parte, la falsa acacia presentó producciones en torno a la mitad que el chopo, en los dos años estudiados de los rebrotes.

\subsection{Análisis de suelos}

La cantidad de materia orgánica (MO) y la disponibilidad de los principales macronutrientes en el suelo no disminuyeron al final de los cuatro años de cultivo (Tab. 2). Al final del periodo estudiado, MO mostró un incremento medio de $0.18 \% \mathrm{y}$ $0.25 \%$ respecto al control, para AF y Rps, respectivamente, aunque no significativo. 
Tabla 2. Análisis de suelos al principio y al final del ensayo (0-20 cm superficiales). * El fósforo se expresa como $\mathrm{P}_{2} \mathrm{O}_{5}$ y se utilizó el método Olsen para su análisis; el potasio se presenta como $\mathrm{K}$ asimilable. $\mathrm{N}$ (N-Kjeldhal), $\mathrm{CE}\left(\mathrm{mS} / \mathrm{cm}, 1: 5\right.$ a $25^{\circ} \mathrm{C}$ ), $\mathrm{pH}$ (al agua, 1:5). MO: materia orgánica. Orig.: suelo original; Orig.+EO: suelo original con aportación de enmienda orgánica; Control; suelo no cultivado y dejado a su libre evolución; R0FA4, R4AF0 y R2AF2: suelos bajo los respectivos tratamientos de cultivo.

\begin{tabular}{lcccccc}
\hline Trat. & Orig. & Orig. + EO & Control & R0AF4 & R4AF0 & R2AF2 \\
\hline Fecha & 2011 & 2011 & 2015 & 2015 & 2015 & 2015 \\
$\mathrm{pH}$ & 8.31 & 8.29 & $8.06 \pm 0.06$ & $8.15 \pm 0.22$ & $8.02 \pm 0.06$ & $8.19 \pm 0.09$ \\
$\mathrm{CE}(\mathrm{mS} / \mathrm{cm})$ & 0.34 & 0.32 & $0.28 \pm 0.03$ & $0.33 \pm 0.04$ & $0.31 \pm 0.01$ & $0.34 \pm 0.06$ \\
$\mathrm{MO}(\% \mathrm{p} / \mathrm{p})$ & 2.98 & 2.91 & $2.88 \pm 0.22$ & $3.06 \pm 0.06$ & $3.13 \pm 0.01$ & $3.07 \pm 0.25$ \\
$\mathrm{C} / \mathrm{N}$ & 10.98 & 10.47 & $9.91 \pm 0.27$ & $11.13 \pm 0.09$ & $10.61 \pm 0.09$ & $10.96 \pm 0.69$ \\
$\mathrm{~N}(\%)$ & 0.157 & 0.161 & $0.168 \pm 0.008$ & $0.159 \pm 0.004$ & $0.171 \pm 0.001$ & $0.162 \pm 0.003$ \\
$\mathrm{P}(\mathrm{mg} / \mathrm{kg})^{*}$ & 35.6 & 36.8 & $38.6 \pm 1.9$ & $37.2 \pm 2.4$ & $41.6 \pm 5.0$ & $40.0 \pm 2.4$ \\
$\mathrm{~K}(\mathrm{mg} / \mathrm{kg})^{*}$ & 558 & 521 & $586 \pm 110$ & $645 \pm 67$ & $534 \pm 49$ & $686 \pm 170$ \\
\hline
\end{tabular}

Asimismo, el índice de relación $\mathrm{C} / \mathrm{N}$ aumentó ligeramente en las parcelas cultivadas respecto al control. Los demás parámetros medidos en el suelo no mostraron empeoramiento en los cuatro años estudiados.

Siguiendo la metodología de Bravo et al. (2007), para los $20 \mathrm{~cm}$ superficiales de suelo muestreado, las cantidades de $\mathrm{MO}$ encontradas equivaldrían a la fijación de 153.2 $\mathrm{t} \mathrm{ha}^{-1}$ de $\mathrm{CO}_{2}$ en el control, que se incrementaría en 9.53 y $13.2 \mathrm{t} \mathrm{ha}^{-1}{\mathrm{de} \mathrm{CO}_{2}}$ en las parcelas de AF y Rps respectivamente. Esto corresponde a incrementos anuales de $\mathrm{CO}_{2}$ fijado en el suelo de 2.38 y $3.30 \mathrm{t} \mathrm{ha}^{-1} \mathrm{año}^{-1}$.

\section{Discusión}

En términos generales y para las dos especies, los tamaños adquiridos por las plantas en altura y diámetro, así como de biomasa producida, están dentro de los rangos propios de estas especies para los primeros años de cultivo (Sixto et al., 2007; Grünewald et al., 2009). No obstante, el tamaño final alcanzado en un turno y la producción de biomasa dependerá de las condiciones edafo-climáticas del lugar de plantación y de la capacidad de adaptación del taxón a estas (Bongarten et al., 1992; Dini-Papanastasi, 2008; Baettig et al., 2010; Sixto et al., 2015). Por ejemplo, estos autores citados han reportado producciones de 3-14 t ha ${ }^{-1}$ año ${ }^{-1}$ para Robinia y $5-22 \mathrm{t} \mathrm{ha}^{-1}$ año ${ }^{-1}$ para clones de Populus. Por tanto, podemos afirmar que las producciones obtenidas en este ensayo se encuentran en un rango medio-alto comparado con otros estudios y plantaciones comerciales. La alta variabilidad encontrada en la Robinia, no solo en crecimiento sino también en manifestación de clorosis foliar, abre una vía de oportuni- 
dades a la selección y mejora genética de la especie para uso en plantaciones comerciales, como así lo han puesto de manifiesto otros autores (Dini-Papanastasi, 2008; Bongarten et al., 1992).

También suele ser habitual, como ocurrió en este estudio, que la producción de biomasa del primer año de plantación sea menor que las producciones de años posteriores, tanto en Populus como en Robinia, y que los rebrotes emitidos tras las cosechas tengan mayor vigor que los crecimientos del primer turno (Dini-Papanastasi, 2008; Baettig et al., 2010; Paris et al., 2011; Quinkenstein et al., 2012; Verlinden et al., 2015). En este estudio, no se constató una mejora de la producción en los tratamientos combinados, por efecto de la fijación de $\mathrm{N}_{2}$ por parte de la falsa acacia, de lo que podría haberse favorecido el chopo. No obstante, parece ser que, para especies arbóreas, el posible trasvase de $\mathrm{N}$ desde la especie fijadora hasta la no fijadora se demora en el tiempo y no comenzaría a observarse los primeros síntomas hasta el tercer año (Bouillet et al., 2008), por lo que queda muy al límite de la duración de este estudio.

A pesar de que el vigor inicial $\left(1^{\circ}\right.$ y $2^{\circ}$ año) del chopo fue mayor que el de la $R o$ binia, viéndose favorecida la producción de biomasa en los tratamientos con mayor porcentaje de chopo, en los años siguientes la falsa acacia alcanzó los niveles productivos del chopo. Incluso lo superó en el cuarto año, cuando las condiciones de humedad edáfica fueron limitadas, lo que justifica su condición de mejor tolerante a la sequía (Grünewald et al., 2009; Sixto et al., 2015). Por otro lado, el menor incremento en biomasa del último año en los bloques no cortados respecto de los cortados puede ser debido a la mayor sensibilidad a la sequía de los primeros en ese período, por tener mayor superficie foliar que los nuevos rebrotes de los segundos. Además, el mayor tamaño en altura y diámetro que alcanzó el chopo sobre la Robinia no se tradujo en mayor biomasa, por las diferencias en densidad básica de la madera $\left(0.39 \mathrm{~kg} \mathrm{dm}^{-3}\right.$ para el clon AF2, frente a $0.75 \mathrm{~kg} \mathrm{dm}^{-3}$ de la Robinia).

Respecto a la evolución del suelo durante los cuatro años que duró el estudio, cabe destacar que no se detectó empeoramiento significativo de ninguno de los parámetros físico-químicos analizados, por lo que no se apreció pérdida de fertilidad o degradación, a pesar del cultivo con especies de crecimiento rápido y de cosechar y extraer biomasa. Más aún, dichos parámetros tienden a permanecer constantes o a mejorar, como en el caso de MO. Las estimaciones hechas en fijación de C (o su correspondencia a $\mathrm{CO}_{2}$ ) están dentro del rango obtenido por otros autores (Quinkenstein et al., 2012; Verlinden et al., 2013), convirtiéndose el cultivo de especies de crecimiento rápido y turno corto en un importante sumidero de $\mathrm{CO}_{2}$, especialmente en el suelo, a pesar del propio cultivo y del aprovechamiento de la biomasa (Berhongaray and Ceulemans, 2015).

\section{Conclusiones}

La supervivencia, los crecimientos y la producción de biomasa se han visto significativamente afectados por las condiciones edafo-climáticas del lugar de planta- 
ción. Las distintas combinaciones de especies ensayadas mostraron ligeras diferencias de producción en distintas fases de cultivo y según el turno de corta. Los tratamientos con mayor proporción del clon de Populus "AF2" mostraron mayor crecimiento en los primeros años de cultivo y tras la corta. Mientras que la Robinia igualó el crecimiento del chopo en turnos más largos, y soportó mejor la sequía. En las condiciones ensayadas, los cultivos forestales de corta duración no afectaron significativamente la fertilidad del suelo en un turno de producción. El empleo de leguminosas en cultivos mixtos contribuiría al incremento de la disponibilidad de $\mathrm{N}$ a medio y largo plazo, pero está por analizar su repercusión en la producción de biomasa en plazos de tiempo más largos que los ensayados en este estudio.

\section{Agradecimientos}

Este trabajo ha sido financiado por el Ministerio de Economía y Competitividad (Plan Nacional I+D+i, ref. AGL2010-16575 y CTQ2013-46804-C2-1-R) y por los fondos FEDER de la UE. Contó con el apoyo de la Diputación Provincial de Granada y del Campus de Excelencia Internacional CEI Cambio.

\section{Bibliografía}

Baettig, R., Yáñez, M., Albornoz, M., 2010. Cultivos dendroenergéticos de híbridos de álamo para la obtención de biocombustibles en Chile: Estado del arte. Bosque. 31(2), 89-99.

Bauhus, J., van Winden, A.P., Nicotra, A.B., 2004. Above-ground interactions and productivity in mixed-species plantations of Acacia mearnsii and Eucalyptus globulus. Can. J. For. Res. 34, 686-694.

Berhongaray, G., Ceulemans, R., 2015. Neglected carbon pools and fluxes in the soil balance of short-rotation woody biomass crops. Biomass and Bioenergy. 73, 62-66. Doi: 10.1016 j.biombioe.2014.12.002.

Bongarten, B.C., Huber, D.A., Apsley, D.K., 1992. Environmental and genetic influences on short-rotation biomass production of black locust (Robinia pseudoacacia L.) in the Georgia Piedmont. For. Ecol. Manage. 55, 315-331.

Bouillet, J.P., Laclau, J.P., Gonçalves, J.L.M., Moreira, M.Z., Trivelin, P.C.O., Jourdan, C., Silva, E.V., Piccolo, M.C., Tsai, S.M., Galiana, A., 2008. Mixed-species plantations of Acacia mangium and Eucalyptus grandis in Brazil. 2: Nitrogen accumulation in the stands and biological N $\mathrm{N}_{2}$ fixation. For. Ecol Manage. 255, 3918-3930. doi:10.1016 /j.foreco.2007.10.050.

Bravo Oviedo, F., Delgado, J. A., Gallardo Lancho, J. F., Bravo Oviedo, J.A., Ruiz-Peinado, R., Merino, A., Montero, G., Cámara, A., Navarro, R., Ordóñez C., Canga, E., 2007. En: El papel de los bosques españoles en la mitigación del cambio climático. Bravo Oviedo, F. (Coord.). Métodos para cuantificar la fijación de $\mathrm{CO}_{2}$ en los sistemas forestales. Fundación gas natural, Barcelona, pp. 65-112.

Consejo de Europa, 2007. Plan de acción del Consejo Europeo (2007-2009). Política Energética para Europa. 7224/1/07 REV1, Bruselas. 
Dini-Papanastasi, O., 2008. Effects of clonal selection on biomass production and quality in Robinia pseudoacacia var. monophylla Carr. For. Ecol Manage. 256, 849-854. doi: 10.1016/j.foreco.2008.05.049.

Fisher, R.F., Binkley, D., 2000. Ecology and Management of Forest Soils. John Wiley \& Sons Inc., New York, pp. 489.

Forrester, D.I., Bauhus, J., Khanna, P.K., 2004. Growth dynamics in a mixed species plantation of Eucalyptus globulus and Acacia mearnsii. For. Ecol. Manage. 193, 81-95.

Forrester, D.I., Bauhus, J., Cowie, A.L., Vanclay, J.K., 2006. Mixed-species plantations of Eucalyptus with nitrogen-fixing trees: a review. For. Ecol. Manage. 233, 211-230.

Geyer, W.A, 2006. Biomass production in the Central Great Plains USA under various coppice regimes. Biomass and Bioenergy. 30, 778-783. doi:10.1016/j.biombioe.2005.08. 002.

Gonçalves, J.L.M., Stape, J.L., Laclau, J.P., Smethurst, P., Gava, J.L., 2004. Silvicultural effects on the productivity and wood quality of eucalypts plantations. For. Ecol. Manage. 193, 45-61.

Grünewald, H., Böhm, C., Quinkenstein, A., Grundmann, P., Eberts, J., Wühlisch, G., 2009. Robinia pseudoacacia L.: A Lesser Known Tree Species for Biomass Production. Bioenerg. Res. 2, 123-133.

IDAE, 2007 Ahorro, eficiencia energética y fertilización nitrogenada. Ahorro y eficiencia energética en la agricultura $n^{\circ}$ 6. Ministerio de Industria, Turismo y Comercio. Madrid, pp.46.

Kelty, M.J., 2006. The role of species mixtures in plantation forestry. For. Ecol. Manage. 233, 195-204.

Laclau, J.P., Ranger, J., Deleporte, P., Nouvellon, Y., Saint-André, L., Marlet, S., Bouillet, J.P., 2005. Nutrient cycling in a clonal stand of Eucalyptus and an adjacent Savanna ecosystem in Congo. 3. Input-output budgets and consequences for the sustainability of the plantations. For. Ecol. Manage. 210, 375-391.

Laclau, J.P., Gonçalves, J.L.M, Silva, E.V., Jourdan C., Cunha, M.C.S., Moreira, M.R., SaintAndré, L., Maquere, V., Nouvellon, Y., Ranger, J., 2008. Mixed-species plantations of Acacia mangium and Eucalyptus grandis in Brazil. Growth dynamics and aboveground net primary production. For. Ecol. Manage. 255, 3905-3917.

Little, K.M., Schumann, A.W., Noble, A.D., 2002. Performance of a Eucalyptus grandis - Eucalyptus camaldulensis hybrid clone as influenced by a cowpea cover-crop. For. Ecol. Manage. 168, 43-52.

Marques, D.B., Martins, P.R.B., Parreira, M.C., Alves, P.L.C.A., 2015. Companion crops upon Eucalyptus urograndis initial development. Bosque. 36(2), 163-170.

McLaughlin, N.B., Hiba, A., Wall, G.J., King, D.J., 2000. Comparison of energy inputs for inorganic fertilizer and manure based corn production. Can. Agric. Eng. 42, 009-017.

Naciones Unidas, 2015. Aprobación del Acuerdo de París (FCCC/CP/2015/L.9). Conferencia de las Partes de la Convención Marco sobre el Cambio Climático, $21^{\text {er }}$ período de sesiones. Disponible en: http://unfccc.int/resource/docs/2015/cop21/spa/109s.pdf. Acceso. 12 de diciembre de 2015.

Paris, P., Mareschi, L., Sabatti, M., Pisanelli, A., Ecosse, A., Nardin, F., Scarascia-Mugnozza, G., 2011. Comparing hybrid Populus clones for SRF across northern Italy after two biennial rotations: Survival, growth and yield. Biomass and Bioenergy. 35(4), 1524-32. doi:10.1016/j.biombioe.2010.12.050. 
Parrotta, J. A., 1999. Productivity, nutrient cycling, and succession in single- and mixedspecies plantations of Casuarina equisetifolia, Eucalyptus robusta, and Leucaena leucocephala in Puerto Rico. For. Ecol. Manage. 124, 45-77.

Pereira, E. L., Santos, S. A. P., Arrobas, M., Patricio, M. S., 2011. Microbial biomass and N mineralization in mixed plantations of broadleaves and nitrogen-fixing species. Forest Systems. 20(3), 516-524.

Quinkenstein, A., Pape, D., Freese, D., Schneider, B.U., Hüttl, R.F., 2012. Biomass, carbon and nitrogen distribution in living woody plant parts of Robinia pseudoacacia L. growing on reclamation sites in the Mining Region of Lower Lusatia (Northeast Germany). International Journal of Forestry Research. Volume 2012, Article ID 891798, 10 pages. doi:10.1155/2012/891798.

Sixto, H., Hernández, M.J., Barrio, M., Carrasco, J., Cañellas, I., 2007. Plantaciones del género Populus para la producción de biomasa con fines energéticos: revisión. Invest. Agrar. Sist. Recur. For. 16(3), 277-294.

Sixto, H., Cañellas, I., Arendonk, J., Ciria, P., Camps, F., Sánchez, M., Sánchez-González, M., 2015. Growth potential of different species and genotypes for biomass production in short rotation in Mediterranean environments. For. Ecol. Manage. 354, 291-299. doi:10.1016/j.foreco.2015.05.038.

Tapias, R., Fernández, M., Alaejos, J., Salvador, L., Alesso, P., González-Duque, J.A., Alfaro, A., López-Baldovin, F., Díaz-Blanco, M.J., 2008. Leguminosas leñosas de rápido crecimiento como cultivos energéticos en el suroeste de la península ibérica. Boletín del CIDEU. 5, 135-147.

Verlinden M.S., Broeckx, L.S., Ceulemans, R., 2015. First vs. second rotation of a poplar short rotation coppice: above-ground biomass productivity and shoot dynamics. Biomass and Bioenergy. 73, 174-185. doi: 10.1016/j.biombioe.2014.12.012.

Verlinden, M.S., Broeckx, L.S., Zona, D., Berhongaray, G., de Groote, T., Camino Serrano, M., Janssens, I.A., Ceulemans, R., 2013. Net ecosystem production and carbon balance of an SRC poplar plantation during its first rotation. Biomass and Bioenergy. 56, 412422. Doi: 10.1016/j.biombioe.2013.05.033.

Voigtlaender, M., Laclau, J.P., Gonçalves, J.L.D., Piccolo, M.D., Moreira, M.Z., Nouvellon, Y., Ranger, J., Bouillet, J.P., 2012. Introducing Acacia mangium trees in Eucalyptus grandis plantations: consequences for soil organic matter stocks and nitrogen mineralization. Plant Soil. 352, 99-111.

Zalesny, R. S., Cunningham, M.W., Hall, R.B., Mirck, J., Rockwood, D.L., Stanturf, J.A., Volk, T.A., 2011. En: Sustainable Production of Fuels, Chemicals, and Fibers from Forest Biomass; ZHU, J. et al. (eds.). Woody Biomass from Short Rotation Energy Crops. ACS Symposium Series; American Chemical Society: Washington, DC., pp. 27-63. 
